\title{
Equilibrium and non-equilibrium structures in complex food systems
}

\author{
Raffaele Mezzenga* \\ Department of Physics, University of Fribourg, Perolles, Fribourg CH-1700, Switzerland \\ Nestlé Research Center, Vers-Chez-Les-Blanc, Lausanne 26 CH-1000, Switzerland
}

\begin{abstract}
The physics ruling the macroscopic behaviour of foods is discussed in a few selected examples in which the equilibrium/nonequilibrium nature can be directly related to the typical length scale of the food structure and the specific interaction strength, the latter describing the decrease in specific free energy (e.g., per mole of species or per single event) when going from a non-equilibrium to the equilibrium configuration. Liquid-crystalline phases in foods, such as those based on self-assembled lipids and water, are discussed as an example of equilibrium system, in which low correlation length scales $(\sim \mathrm{nm})$ enable very-short times to re-organize molecules into equilibrium configurations. High internal phase emulsions and dry foams where length scales are very large $\left(\sim 10^{1}-10^{4} \mu \mathrm{m}\right)$, are discussed as a typical example of non-equilibrium system, which however, can be stable for long times owing to the high free energy barrier and the large correlation lengths. Finally double emulsion systems are considered as an example where both interaction strength and correlation lengths have intermediate values. This allows tuning morphologies and making equilibrium behaviour to prevail over non-equilibrium by suitably tailoring the energetic interactions. In particular the case of osmotic pressure in the water phase of water/oil/water double emulsions is discussed as a viable route to control interaction strength in complex food systems.
\end{abstract}

Keywords: Emulsions; Double emulsions; High internal phase emulsions (HIPE); Protein networks; Gels; Interfaces; Liquid crystals; Mesophases; Self-assembly

\section{Introduction}

Although most processed foods are encountered far from equilibrium, understanding and controlling equilibrium food structures is an important issue because it sets a general guideline for rationally designing structure-properties relationship. Non-equilibrium or "metastable" morphologies, on the other hand, which are the great majority of available foods, paradoxically, can be made stable for very long times. How can this be achieved, what are the driving forces towards equilibrium or nonequilibrium structures?

When investigating and modelling the behaviour of foods one can use the same experimental techniques and approach used to design morphology in soft condensed matter,

\footnotetext{
*Department of Physics, University of Fribourg, Perolles, Fribourg CH-1700, Switzerland. Tel.: +4126300 9066; fax: + 41263009747.

E-mail addresses: raffaele.mezzenga@unifr.ch,

raffaele.mezzenga@rdls.nestle.com (R. Mezzenga).
}

although the level of complexity encountered in foods generally goes well beyond that of typical model systems studied in soft condensed matter physics (Mezzenga, Schurtenberger, Burbidge, \& Michel, 2005). The source of complexity in food systems responsible for non-equilibrium behaviour can be considered three-fold, as schematically illustrated in Fig. 1. Firstly, the aggregation state in which foods can be found varies over a broad range. In the case of macromolecular systems, foods can be found in dilute polymer solutions, semi-dilute solutions and glassy amorphous or crystalline polysaccharide matrices. Whereas, for dilute and semi-dilute solutions equilibrium may be easily attained, owing to the presence of entanglements and hydrogen bonding, glassy matrices are often found in nonequilibrium state (Kilburn et al., 2004). Analogously, in the case of colloidal dispersions, by increasing the volume fraction of the colloidal dispersion one can pass from a viscous dispersion, to a gel, and finally obtain a glassy (or crystalline) solid (Pusey \& van Megen, 1986). Reversible phase transitions can still be observed here, provided 


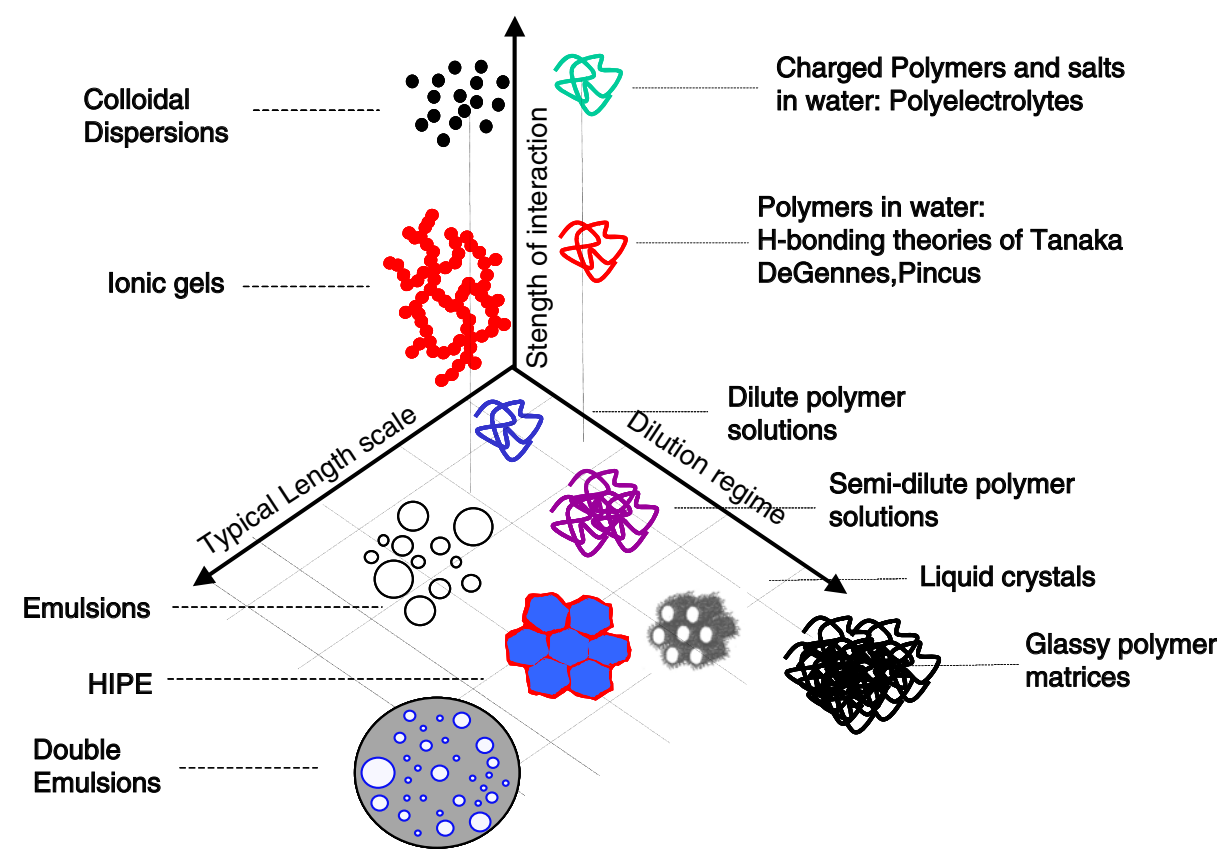

Fig. 1. The nature of complexity in physics of foods.

that the inter-particle distance is maintained large enough to keep away the system from the attractive irreversible van der Waals potential well. Another factor adding complexity to foods is the variable potential, or strength of interaction, by which food components interact between each other. A detailed physical description of neutral food-grade polymers in water, for example, has to account for the polymer-water hydrogen bonding interactions, which being on the order of one or several $k T$ per bond, lead to a non-negligible contribution in the total free energy/macromolecule (Matsuyama \& Tanaka, 1990). Models specifically developed for water-soluble synthetic polymers, appear to be suitable for this specific problem (Bekiranov, Bruinsma, \& Pincus, 1997; De Gennes, 1991; Matsuyama \& Tanaka, 1990). On the other hand, many food polymers, such as various polysaccharides are electrically charged, and for these strongly interacting systems, poly-electrolyte theories have to be used (Jones, Goodall, Cutler, \& Norton, 1987). The variation of strength of interactions has also dramatic consequences in another large family of foods: the colloidal dispersions. The phase diagram of food and biological colloidal dispersions, in terms of interaction strength and volume fraction is so rich that recently a number of works have been devoted to this topic (Denton \& Schmidt, 2005; Dickinson \& Krishna, 2001; Stradner et al., 2004). For example, sol-gel and fluid-solid transitions in a colloidal protein dispersion can be tuned by only changing the interaction strength, for example with the $\mathrm{pH}$ or ionic strength, and keeping unaltered the volume fraction of the protein. On the other hand, complex phenomena such as melting of a glassy food colloidal dispersion can also be observed and tailored, if for example weak short range attractive forces, such as the case of polysaccharide-controlled depletion forces, are introduced in order to position the system close to the re-entrant region of the force-composition state diagram (Dawson, 2002; Pham et al., 2002). A final source of intrinsic complexity in food systems is the typical length scale at which foods are structured. Because this length scale can span as much as six orders of magnitude (from few $\mathrm{nm}$ to several $\mathrm{mm}$ ), clearly the physics has to be ruled by different driving forces at different length scales, which will make either the equilibrium (at small length scales) or nonequilibrium (at large length scales) to prevail. Therefore, in order to make possible morphological changes in a system far from thermodynamic equilibrium, entities that can react or aggregate have to encounter each other, which depending on distances or transport rates may require very long times. Furthermore, whenever this happens an activation free energy will have to be overcome in order to allow interaction among compounds and thus, reorganization of the typical structures. In the present paper focus will be given to "dense" viscoelastic foods and the conditions for observing truly equilibrium or non-equilibrium structures will be discussed in terms of the characteristic length scale and typical interaction strength of the system.

\section{Intermediate interaction strength and length scale: Double emulsions}

Double and multiple emulsions constitute an attracting system for controlled delivery of specific components and ingredients, both in hydrophilic and hydrophobic environment (Garti \& Bisperink, 1998; McClements \& Demetriades, 1998). Their use in industrial applications has been, however, severely hindered by the short 
lifetime related to the many possible forms of instabilities these systems can suffer (Garti, 1998; Sela, Magdassi, \& Garti, 1995). While these instabilities have been discussed more extensively elsewhere for the water/oil/water $(\mathrm{W} / \mathrm{O} / \mathrm{W})$ system, it will be stressed here that three major forms of instabilities may occur: (i) coalescence of the primary emulsion internal droplet phase (in the case of $\mathrm{W} / \mathrm{O} / \mathrm{W}$ double emulsions, the internal water droplet phase), (ii) coalescence of the secondary emulsion outer droplet phase (in the case of $\mathrm{W} / \mathrm{O} / \mathrm{W}$ double emulsions, the external oil droplet phase) and (ii) suppression, coalescence or swelling of the internal droplets due to unbalanced osmotic-Laplace pressures. While the first two effects may be controlled to a very good extent by efficient design of the surfactants used to stabilize the droplets against coalescence via an absorbed monolayer, the Laplace pressure always needs to be equilibrated by an opposite osmotic pressure. In a pure $\mathrm{W} / \mathrm{O} / \mathrm{W}$ double emulsion for example, the Laplace pressure associated with the small water droplets will let water diffusing from the inner to the outer phase. A common trick used to reduce this effect is to dissolve a low molecular weight compound into the internal droplet phase, such as salt or sugar, able to efficiently decrease the chemical potential of water and thus, to induce an osmotic pressure gradient opposing the drift due to the Laplace pressure. Because low molecular weight compounds may be weakly soluble also in the oil phase, double emulsions may still be unstable at long time scales.

The use of polysaccharides of high molecular weight has been proposed as a technique to tune the osmotic pressure in a more controlled way, since both concentration and molecular weight of the polysaccharide can be used to tailor the chemical potential of water, and thus the osmotic pressure, without diffusion (for high enough molecular weights) of the host molecules between the inner and outer water phases (Mezzenga, Folmer, \& Hughes, 2004). Furthermore, by using suitable molecular weights on the external water phase, one has also the possibility of inverting the flow of water from the inner to the outer phase, leading to shrinking of the $\mathrm{W} / \mathrm{O} / \mathrm{W}$ double emulsions. Because the Laplace and osmotic pressures have to be fully balanced at the equilibrium, one can actually predict the final morphology of multiple emulsions by equalizing the contribution of Laplace pressure with welldefined models for osmotic pressure in water-polymer mixtures.

For a single internal water droplet this balance is expressed by

$\Delta \Pi=\frac{2 \gamma}{r}$,

where $\Delta \Pi$ is the osmotic pressure difference between the external and internal water phase induced by the dissolved polysaccharide, and $r$ and $\gamma$ are the interfacial tension and the radius of the internal water droplet, respectively.
For polymer-water solution, the osmotic pressure difference can be expressed as

$\Delta \Pi=\frac{R T}{v_{0}}\left[\frac{\phi_{1}}{N}+\ln \left(\frac{1}{1-\phi_{1}}\right)-\phi_{1}-\chi \phi_{1}^{2}\right]$,

where $R$ is the ideal gas constant, $T$ the absolute temperature, $v_{0}$ the molar volume of water, $N$ the degree of polymerisation of the water-soluble polymer, $\chi$ the Flory-Huggins interaction parameter between polymer and water, and $\phi_{1}$ represents the volume fraction of the polymer in the internal droplet. By entering Eq. (2) into Eq. (1) and solving with respect to $r$ one can work out the equilibrium radius of the internal water droplet, and extending this result to the internal water particle size distribution, one can predict the final size distribution of double emulsion droplets.

In this way, provided that interfaces are well stabilized, true equilibrium morphology can be designed, predicted and observed (Mezzenga et al., 2004). The parameters one can change to design and control the morphology are the typical length scale at which the inner emulsion is structured, that is, the average diameter size, as well as the interaction strength, e.g. the nature of the polymer dissolved into the water phase. Lowering the average diameter size results in increasing the Laplace pressure and thus the amount of water, which needs to diffuse from the outer to the inner phase. Correspondingly, interaction strength can also be modified to alter the morphology of multiple emulsions. By introducing poly-electrolytes such as charged polysaccharides, rather than neutral polymers, allows increasing more efficiently the osmotic pressure gradient and thus, it results in effects similar to those observed when the average diameter size of the internal droplet is decreased (Folmer, 2004). Finally, one can finely tune the osmotic pressure induced by polyelectrolytes by the presence of salts, which, by their effect of screening charges of polyelectrolytes will also moderate the chemical potential change, and therefore osmotic pressure. Fig. 2 sketches the lines of though of controlling the morphology of $\mathrm{W} / \mathrm{O} / \mathrm{W}$ double emulsions by tailoring the osmotic pressure.

Tuning the interaction strength and structure length scale has effects not only on the morphology, but also on the characteristic relaxation time needed to reach equilibrium. Although no analytical models have been presented

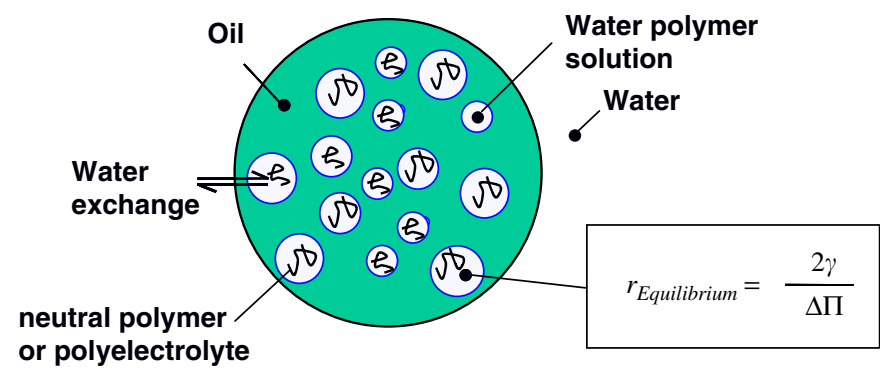

Fig. 2. Control of $\mathrm{W} / \mathrm{O} / \mathrm{W}$ morphologies via tuneable osmotic pressure. 
yet to predict the typical relaxation time of double emulsions, experimental data indicate that times of $1-24 \mathrm{~h}$ are typically needed to achieve osmotic equilibrium (Folmer, 2004; Mezzenga et al., 2004). Thus the conditions for observing equilibrium structures really depend on the difference between the typical relaxation time and the time of observation. Clearly, it is possible to decrease the neat driving force for water diffusion to such an extent that equilibrium will be reached at very long timescales, possibly longer than the observation time. In general this method relies on designing a specific morphology at the equilibrium, in such a way that major changes in shape or particle size distribution are avoided after the $\mathrm{W} / \mathrm{O} / \mathrm{W}$ has been produced.

Other techniques have been proposed to increase the lifetime of double emulsions, which however, go in the opposite direction than designing a system close to equilibrium. One of these techniques makes use of an oil phase, which can be crystallized by temperature changes (Guery et al., 2006). In such a case, the first and second emulsification processes are realized at high temperature so as to have a liquid $\mathrm{W} / \mathrm{O} / \mathrm{W}$ double emulsion system. At some point, however, temperature is rapidly quenched in order to let the majority of the oil phase crystallize, thus "freezing" the morphology of the double emulsion into a well-defined configuration. Obviously, if the process is rapid enough, the frozen morphology at low temperature will correspond to a non-equilibrium morphology. Nevertheless, the presence of a phase which consists mostly of solid crystalline fat is sufficient to suppress virtually any diffusion of the water phase, as well as any coalescence among inner or outer droplets, leading to a relaxation time which is virtually infinite.

\section{High interaction strength and intermediate length scales: Dry foams and high internal phase emulsions (HIPE)}

HIPE, that is emulsions in which the dispersed phase is exceeding 0.74 in volume fraction, are a typical example of non-equilibrium structures because the interfacial free energy associated with the large interfacial area is maximized rather than being minimized (Lissant, 1966; Lissant \& Mayhan, 1973). In such a case, the system is not at its minimum total free energy and thus, far from equilibrium. When low molecular weight surfactants are used to stabilize interfaces, such as lecithin, at long timescales the great majority of HIPE emulsions will tend to coalesce to inverted simple emulsions, although a careful design of either phase or of the specific processing route may greatly enhance their long-term stability (Lissant \& Mayhan, 1973). The route selected to design specific HIPE and the resulting morphology in particular, account to a great extent for the instabilities characteristic of these particular blends. For example, the size distribution of the droplet forming the disperse phase is a critical parameter. Very poly-disperse emulsions have the capability of rearranging their morphology in such a way as to locate the smallest droplets at the interstitial spaces left unoccupied by the largest droplets. Thus, virtually any volume fraction of the dispersed phase can possibly be attained without deforming the droplets and maintaining their spherical shape. On the other hand, mono-disperse droplet systems, or HIPE with a very narrow particle size distribution will undergo a sphere-to-polyhedron transition of the particle shape when volume fraction beyond 0.74 is reached. If one draws a direct analogy with mono-disperse dry foams, where the dispersed phase is replaced by air, the deformation of droplets is accompanied with an increase of the interfacial area of the order of $10 \%$, as in the case of Kelvin foams (Weaire \& Phelan, 1994).

If the surfactant is used in such a quantity to just saturate the original interface, the deformation will be accompanied by a dilution of the surfactant interfacial density, an increase in interfacial tension and thus, and an increase of the driving force towards coalescence (Romoscanu \& Mezzenga, 2005). The corresponding increase in interfacial tension with increased area, which is virtually the same for each cell in a mono-disperse droplet system, can be calculated by (Romoscanu \& Mezzenga, 2005)

$$
\begin{aligned}
\sigma_{A}= & \sigma_{0}+\int_{A_{0}}^{A} E_{D} \mathrm{~d} \ln (A)=\sigma_{0}+E_{D} \ln \left(\frac{A}{A_{0}}\right) \cong \sigma_{0} \\
& +0.1 E_{D},
\end{aligned}
$$

where $\sigma_{A}$ is the interfacial tension of the strained interface of area $A, \sigma_{0}$ the interfacial tension of the unperturbed surface and $E_{D}=\mathrm{d} \sigma / \mathrm{d} \ln A$ is the interfacial elasticity, measured by tensiometric experiments. This effect is, however, generally overcome by stabilizing interfaces with small excess of surfactants, which can be re-adsorbed during the interfacial expansion associated with the sphereto-polyhedron transition. Yet, issues of kinetics may then become central in preserving the general morphology of the HIPE.

About a decade ago, Kunieda and co-workers have reported first, examples of water-in-oil HIPE which form spontaneously and which are stable over long times within large regions of the temperature/composition phase diagram (Kunieda, Fukui, Uchiyama, \& Solans, 1996). In their work, they used water, decane as hydrophobic phase and tetraethylene glycol dodecyl ether as surfactant $\left(\mathrm{C}_{12} \mathrm{EO}_{4}\right)$. This surfactant alone or with small amounts of water forms lamellar phase. Thus, water-in-oil HIPE were obtained by progressively swelling the lamellar phase with water driving it into a HIPE. Although rapid changes in temperature were needed to enter the stability phase diagram regions without dwelling in those regions more sensitive to Oswald ripening, the results of Kunieda's work suggested thermodynamically stable HIPE being possible. Although these results may appear surprising at a first sight, the achievement of stable HIPE was mostly depending on the suitable design of the interfacial oligomeric surfactant. 
The possibility of designing theoretically the most suitable molecular architecture of macromolecular surfactants in order to achieve thermodynamically stable HIPE has been tackled by using self-consistent field models (Mezzenga, Fredrickson, \& Kramer, 2003). By systematically changing the hydrophobic/hydrophilic balance of the surfactant, it was demonstrated that equilibrium morphologies can be achieved in which the majority phase is stable in the form of a discrete dispersed phase and the minority phase is stable as a continuous phase. Although the maximum possible volume fraction of the dispersed phase was of the order of $60 \%$, theoretically this value can still be increased by raising the chemical potential of the continuous phase with respect to that of the macromolecular surfactant compatible block. In macromolecular systems this corresponds to the so-called conditions of "wet brush", i.e., a monolayer in which the block compatible with the continuous phase is greatly swollen by the molecules of the minority phase. This can be achieved, for example, by simply decreasing the molecular weight of the continuous phase or increasing the length of the compatible surfactant block.

Nevertheless, in foods, the conditions for optimising the molecular architecture of the surfactants are severely restricted by the need of preserving their food-grade nature, and in practice very limited types of macromolecules are available to stabilize interfaces, such as proteins for oil-in water emulsions or ricinoleic acid (PGPR) for water-in-oil emulsions. Therefore, other strategies may be appealing to stabilize HIPE in foods. Taking inspiration from the synthetic analogues, where poly-HIPE, have been widely developed and employed, HIPE where both the continuous and dispersed phase are polymerised by a liquid HIPE containing polymerisable units in both phases
(Cameron \& Barbetta, 2000; Kempe \& Barany, 1996), or solidification of either phase or both phases in Food HIPE appear as a viable and promising route. This could be achieved, for example, by using crystallisable oils for the hydrophobic phase and a gelling water-pectin or watercarrageenan solutions for the hydrophilic phase.

Once again, Romoscanu and Mezzenga (2006) have taken inspiration from synthetic polymer and colloidal physics (Mezzenga, Ruokolainen, Fredrickson, \& Kramer, 2003; Mezzenga, Ruokolainen, Fredrickson, Kramer, Moses et al., 2003; Mezzenga, Ruokolainen, \& Hexemer, 2003) to design very stable and fully reversible food grade in water-HIPE by self-assembly of protein-coated monodisperse oil droplets. The new strategy in their work is the cross-linking of the interfacial protein layer while preserving the food grade nature of the emulsion. In practice this can be realized by cross-linking the protein macromolecules by thermal, enzymatic or chemical processes, in a very dilute dispersion of protein-coated oil droplets in water, so that bridging cross-links between protein monolayers of different droplets are avoided. Then, the emulsion template forms a colloidal crystal upon evaporation of water, and when water has been removed down to an extent of 0.26 , the droplets start to deform to undertake the spherepolyhedron transition.

However, because the droplet surface is now crosslinked, coalescence is completely suppressed. Thus water can be removed to virtually nil content, leaving the HIPE in the form of a protein-percolating network of surfaces dispersed in an oil matrix, corresponding to an oil volume fraction as high as $99.9 \%$ (see Fig. 3). Besides the surprising effect of dispersing proteins in oil and providing low molecular weight oils with viscoelastic properties, this method shows to be fully reversible, allowing a full

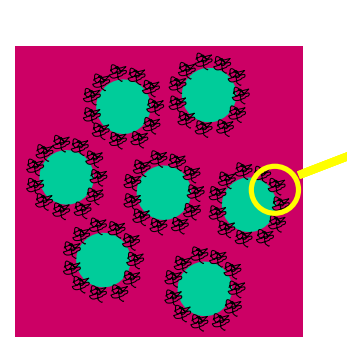

(A)

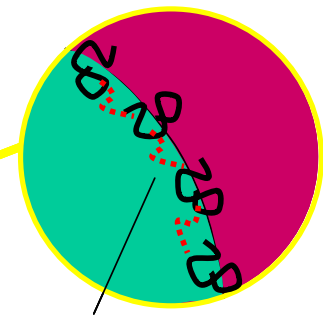

Crosslink Protein Film
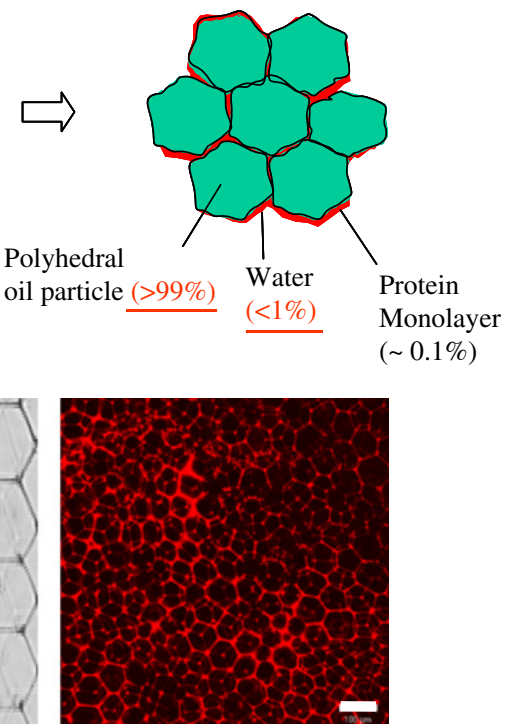

$(\sim 0.1 \%)$

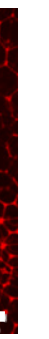

(B)
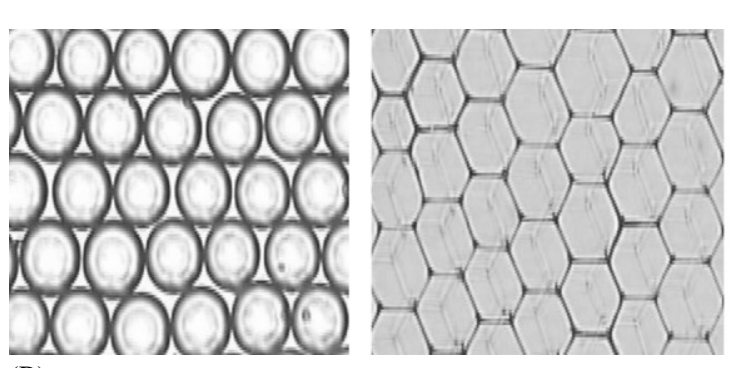

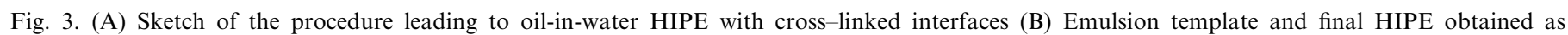
highlighted in (A). Scale bar is $100 \mu \mathrm{m}$, readapted from Romoscanu and Mezzenga (2006). 
re-hydration of the HIPE back to the original emulsion template, thus recovering the original particle size distribution. This example clearly shows that by tuning interaction strength, even non-thermodynamic equilibrium morphologies (the continuous phase is as low as $0.1 \%$ in volume fraction!) can undergo fully reversible cyclic processes.

\section{Liquid-crystalline phases in foods}

Liquid-crystalline phases encountered in foods are, for example, those obtained by the self-assembly of lipids and water (Krog, 1990). These systems form a large spectrum on mesophases, most common among which are lamellar, hexagonal, isotropic micellar, cubic micellar and bicontinuous cubic. All of these mesophases are characterized by both lyotropic and thermotropic behaviour. The typical interaction strength ruling the phase behaviour of these systems is the enthalpy of mixing of the hydrophobic and hydrophilic domains, which is high, and is indeed responsible for the macroscopic immiscibility of water and oil. At room temperature this enthalpy of mixing has been evaluated by the residual partitioning of homologue series of alkanes in water and quantified to be of the order of $\chi=3$, where $\chi$ is the Flory-Higgins interaction parameter between hydrocarbon chains and water, which is equivalent to $3 k T$ per pair molecules of different chemical species (Mezzenga, Meyer et al., 2005; Mezzenga, Schurtenberger et al., 2005). Because liquid-crystalline phases such as those based on self-assembly of water and lipids are typically organized within a characteristic period of 10 or less nanometers, the interaction strength towards segregation is relatively strong over very short length scales. This has mostly two effects: the first is the minimization of perturbation effects on the structure, which typically arise from thermal fluctuations. Secondly, molecules have to re-arrange over very short distances in order to minimize their free energy. Thus, provided that transport phenomena occur within short time scales, the conditions for achieving thermodynamic equilibrium are gathered together.

Kinetics and transport phenomena for each liquidcrystalline phase can be quantified by measuring the typical relaxation time. Although relaxation phenomena in liquidcrystalline phases have been investigated with various experimental techniques, numerical simulations and theoretically (De Gennes, 1975; Jones \& McLeish, 1995; Jones \& McLeish, 1999; Lauger, Robertson, Frank, \& Fuller, 1996; Shinoda, Namiki, \& Okazaki, 1997), the most widely used approach to quantify the typical relaxation times is rheology (Ferry, 1980; Radiman, Toprakcioglu, \& McLeish, 1994; Montalvo, Valiente, \& Rodenas, 1996). In particular, the longest relaxation time, which is the essential parameter to describe the viscoelastic behaviour, can simply be extracted by the inverse of the frequency at which the storage and loss shear moduli intersect in a frequency scan shear rheology experiment. Common relaxation times extracted by this method for various lipid-based liquid-crystalline phases typically range between 0.1 and $10 \mathrm{~s}$. Yet, one should be aware that the picture of relaxation phenomena based on a single relaxation time is somewhat simplistic. As recognized already in literature, liquid-crystalline phases in selfassembled lipids have a viscoelastic behaviour characterized by several relaxation mechanisms, and thus, by several relaxation times. Thus, in order to account for their complex viscoelastic behaviour, several methods have been proposed, based either on multiple Maxwell models, or on discrete relaxation spectra (Mezzenga, Meyer et al., 2005; Mezzenga, Schurtenberger et al., 2005). As a result, various relaxation times have been identified and argued to be characteristic of specific interfaces or constrained phases, without, however, changing considerably the conclusions which can be drawn by considering the longest relaxation time directly provided by storage and loss moduli crossover. Whatever method is used to extract the relaxation time, its low value in self-assembled liquid-crystalline phases (compared to many other examples of nanostructured soft condensed materials), identifies very fast relaxations, which are consistent with the lack of entanglements and connectivity of mesophases. Together with the short characteristic length scales and high energetic driving forces towards segregation, the fast relaxation phenomena allow understanding of why self-assembled lipids in water are most commonly observed at the equilibrium.

Despite these arguments, metastable mesophases can still be observed in self-assembled lipid-water systems. This occurs, for example, when the viscosity is too high to slow down transport phenomena, and thus, relaxation mechanisms. One example is the micellar cubic ( $\mathrm{Fd} 3 \mathrm{~m})$-hexagonal transition occurring in monoglycerides-water-tetradecane systems upon increase of temperature (Yaghmur et al., 2006). Because diffusion is greatly delayed in the highly viscous $\mathrm{Fd} 3 \mathrm{~m}$ phase, the hexagonal phase may not be attained within standard measuring times upon increasing temperatures. On the other hand, the hexagonal-cubic transition is easily achieved within short times when temperature is decreased because the starting conditions correspond, in this case, to the less viscous hexagonal phase.

Provided that one is observing and measuring structures at real thermodynamic equilibrium, mesophases of selfassembled liquid-crystalline lipid-water mixtures can, in principle, be modelled and predicted within mean-field theories.

According to this approach, each lipid chain experiences a mean field generated by all the surrounding lipid chains and water. The free energy of each chain can then be calculated based on existing model describing the nature of the chain. For example, the harmonic stretching energy associated with the interactions of segments and nonbonded interactions, can be associated with a mesoscopic particle model (similarly to block copolymer systems) which allows writing the discrete partition function (and thus the Hamiltonian) of the lipid chains in the surround- 
ing medium. Then, by introducing delta-Dirac operator of the density of each chemical component, a mathematical procedure known as Hubbard-Stratonovich transformation (Fredrickson, Ganesan, \& Drolet, 2002), this particlebased model is transformed into a continuous field problem. Finally, equilibrium configurations are obtained by equalizing to zero the first derivative of the Hamiltonian with respect to the density of each component (in this case water and lipid), which can be solved by either finedifference or fine-element methods. The central assumption of this method is that any specific mesoscopic equilibrium configuration corresponds to local minima of the total free energy of mixing.

Self-consistent field theory (SCFT) simulations based on the mean-field approximation, have been successfully applied to various polymer systems (Edwards, 1965; Fredrickson, 2006) and in particular to block copolymer mesophases, which can be viewed as the synthetic analogues of self-assembled lipid liquid crystals (Matsen, 1995; Matsen \& Schick, 1994). The major difficulties arising when applying SCFT theory to self-assembled lipids at equilibrium are mostly two folds. Firstly the lipid chains are much shorter than block copolymer chains analogues, and the partition function used for polymer Gaussian chains cannot be used to properly describe single lipid molecules. Secondly, the nature of interaction between the selective solvent (water) and the hydrophilic surfactant block (the polar head) is complicated by the presence of hydrogen bonding interactions, which being disrupted by temperature, are mostly responsible for the lyotropic behaviour of lipid-water systems. Pioneering work on the use of SCFT as a tool to describe self-assembled lipids at equilibrium has been carried out by Schick and co-workers (Li \& Schick, 2001; Muller \& Schick, 1998), which have introduced a rotational isomeric state (RIS) model to treat the specificity of lipid chains. In such an approach the lipid chains remain essentially a random walk with Gaussian distribution, but conformational energy of the configuration of subsequent monomers can be used to determine the persistence length of the chain, and thus the entropic contribution to the free energy. Further effort towards a SCFT description of self-assembled lipids in water, has been taken by Fredrickson and co-workers who have been able to model the most common mesophases observed in water-lipid mixtures, and more specifically the lamellar phase, the reverted columnar hexagonal phase, as well as
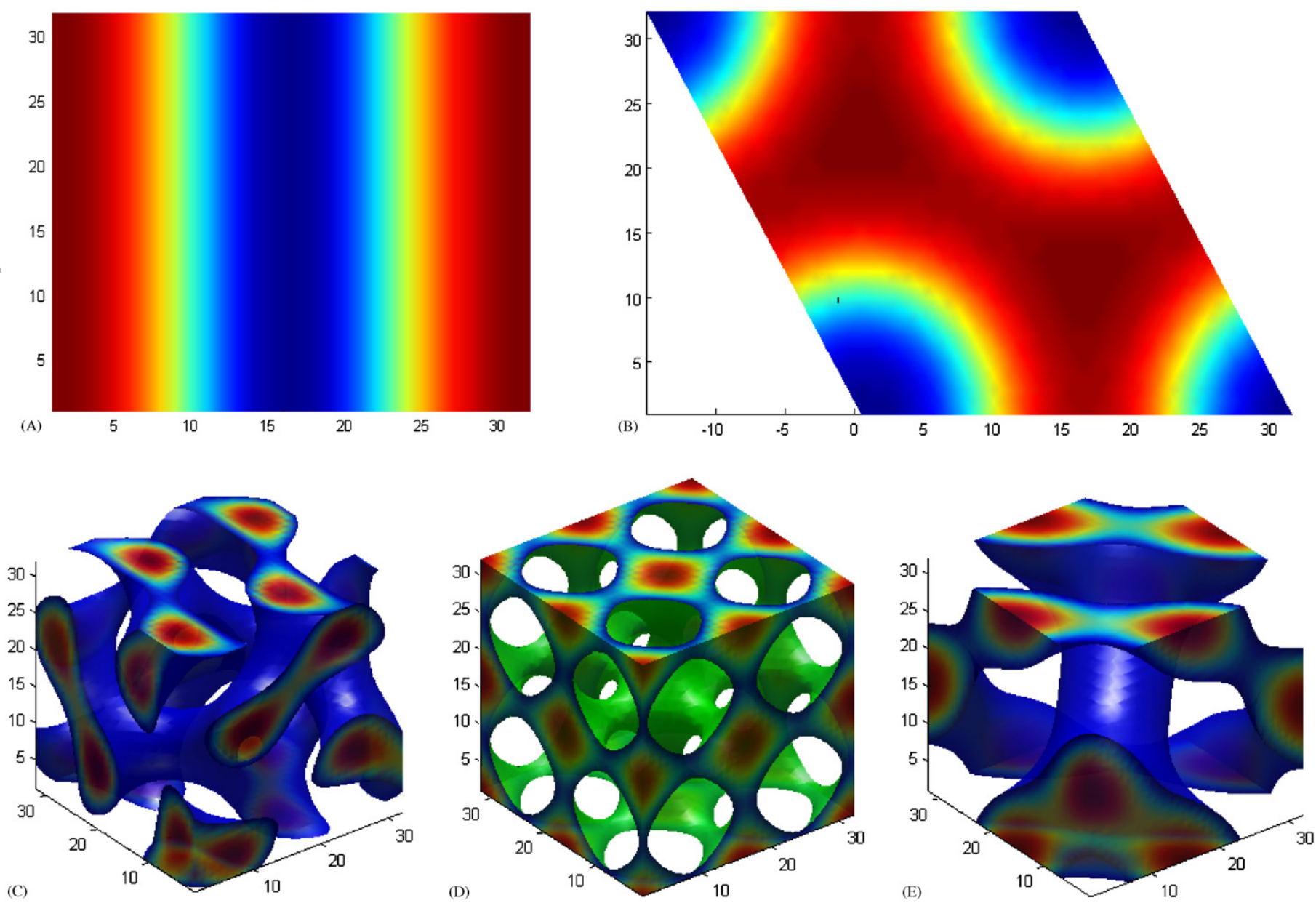

Fig. 4. Lamellar (A), inverted columnar hexagonal (B), inverted double gyroid Ia3d cubic phase (C), inverted primitive Im3m cubic phase (D) and inverted Pn3m double diamond cubic phase (E) as obtained by SCFT simulations (Mezzenga et al., 2006). 
the three major classes of inverted bi-continuous cubic phases (Ia3d, Pn3m, Im3m) (Mezzenga, Lee, \& Fredrickson, 2006) (see Fig. 4). Because of the rough assumptions made in their model, the quantitative agreement with real phase diagrams remains, at present, very limited. Yet, for any specific lipid-water system, their approach not only allows predicting liquid-crystalline phases at equilibrium, but also the relevant topological parameters at a given temperature and composition, such as the radius of water channels or width of lipid bi-layers, thus providing the basis for a rational design of multi-component complex liquid-crystalline foods at equilibrium.

\section{Outlook and conclusion}

A few selected examples of food systems, such as HIPE, double emulsions and self-assembled lipid-water mesophases were discussed as examples of complex systems in which, the equilibrium/non-equilibrium nature can be directly related to the typical length scale of the food structure and the total free energy of the system. Liquidcrystalline phases in foods, such as those based on selfassembled lipids and water, constitute a perfect example of a complex food at equilibrium. The low correlation length scales $(\sim \mathrm{nm})$ in this system, together with their low relaxation time $(\sim 1-10 \mathrm{~s})$ enable very short times to reorganize molecules within small distances and thus to achieve equilibrium configurations. HIPE and dry foams where length scales are very large $\left(\sim 10^{1}-10^{4} \mu \mathrm{m}\right)$, and where the cross-linking of the interfaces can increase the relaxation time to virtually infinite values, are discussed as a typical example of non-equilibrium, yet stable system, characterized by the high free energy connected with very large interfaces. Finally $\mathrm{W} / \mathrm{O} / \mathrm{W}$ double emulsion systems are discussed, in which the relaxation time, taken as the time needed to achieve dynamic equilibrium between the Laplace pressure of internal droplets and the difference in osmotic pressure between internal and external water phases, can be tailored by the presence of hydrophilic compounds dissolved in the water phases. In particular, by using neutral polymers or polyelectrolytes as hydrophilic agents to affect the chemical potential of the two water phases, one can tune their relaxation time ( hours) and design the final equilibrium morphologies of $\mathrm{W} / \mathrm{O} / \mathrm{W}$ emulsions. Other routes are also discussed to stabilize non-equilibrium $\mathrm{W} / \mathrm{O} / \mathrm{W}$ emulsions by solidifying the oil phase.

In summary, in order to achieve truly equilibrium structures, three conditions need to be met: (i) short length scales favouring local rearrangement without the need of long mean displacements, (ii) high free energy driving forces towards segregation, which allows minimizing thermal fluctuations and can generate morphology changes fulfilling total free energy minimization, (iii) short relaxation times, which allows attaining equilibrium configurations within observable time scales. The lack of any of the above conditions can be responsible for non-equilibrium structures of foods.

\section{References}

Bekiranov, S., Bruinsma, R., \& Pincus, P. (1997). Solution behavior of polyethylene oxide in water as a function of temperature and pressure. Physical Review E, 55(1), 577-585.

Cameron, N. R., \& Barbetta, A. (2000). The influence of porogen type on the porosity, surface area and morphology of poly(divinylbenzene) polyHIPE foams. Journal of Materials Chemistry, 10(11), 2466-2472.

Dawson, K. A. (2002). The glass paradigm for colloidal glasses, gels, and other arrested states driven by attractive interactions. Current Opinion in Colloid and Interface Science, 7(3-4), 218-227.

De Gennes, P. G. (1975). The physics of liquid crystals. London: Oxford University Press.

De Gennes, P. G. (1991). A second type of phase separation in polymer solutions. Comptes Rendus de l'Académie des Sciences II, 313(10), $1117-1122$

Denton, A. R., \& Schmidt, M. (2005). Mixtures of charged colloid and neutral polymer: Influence of electrostatic interactions on demixing and interfacial tension. Journal of Chemical Physics, 122(24) Art. no. 244911.

Dickinson, E., \& Krishna, S. (2001). Aggregation in a concentrated model protein system: A mesoscopic simulation of beta-casein self-assembly. Food Hydrocolloids, 15(2), 107-115.

Edwards, S. F. (1965). The statistical mechanics of polymers with excluded volumes. Proceedings of the Physical Society, 85(546P), 613-624.

Ferry, J. D. (1980). Viscoelastic properties of polymers. New York: Wiley. Folmer, B.M. (2004). Unpublished results.

Fredrickson, G. H. (2006). The equilibrium theory of inhomogeneous polymers. London: Oxford University Press.

Fredrickson, G. H., Ganesan, V., \& Drolet, F. (2002). Field-theoretic computer simulation methods for polymers and complex fluids. Macromolecules, 35(1), 16-39.

Garti, N. (1998). A new approach to improved stability and controlled release in double emulsions, by the use of graft-comb polymeric amphiphiles. Acta Polymerica, 49(10-11), 606-616.

Garti, N., \& Bisperink, C. (1998). Double emulsions: Progress and applications. Current Opinion in Colloid and Interface Science, 3(6), $657-667$.

Guery, J., Betrand, E., Rouzeau, C., Levitz, P., Weitz, D.A., \& Bibette, J. (2006). Irreversible shear-activated aggregation in non-brownian suspensions. Physical Review Letters, in press.

Jones, J. L., \& McLeish, T. C. B. (1995). Rheological response of surfactants cubic phases. Langmuir, 11(3), 785-792.

Jones, J. L., \& McLeish, T. C. B. (1999). Concentration fluctuations in surfactant cubic phases: Theory, rheology, and light scattering. Langmuir, 15(22), 7495-7503.

Jones, S. A., Goodall, D. M., Cutler, A. N., \& Norton, I. T. (1987). Application of conductivity studies and polyelectrolytes theory to the conformation and order-disorder transition of xanthan polysaccharide. European Biophysics Journal with Biophysics Letters, 15(3), 185-191.

Kempe, M., \& Barany, G. (1996). A novel family of highly cross-linked polymeric supports for solid-phase peptide synthesis. Journal of the American Chemical Society, 118(30), 7083-7093.

Kilburn, D., Claude, J., Mezzenga, R., Dlubek, G., Alam, A., \& Ubbink, J. (2004). Water in glassy carbohydrates: Opening it up at the nanolevel. Journal of Physical Chemistry B, 108(33), 12436-12441.

Krog, N. J. (1990). In S. E. Friberg, \& K. Larsson (Eds.), Food Emulsions (pp. 141-188). New York: Marcel Dekker.

Kunieda, H., Fukui, Y., Uchiyama, H., \& Solans, C. (1996). Spontaneous formation of highly concentrated water-in-oil emulsions (gel-emulsions). Langmuir, 12(9), 2136-2140.

Lauger, J., Robertson, C. R., Frank, C. W., \& Fuller, G. G. (1996). Deformation and relaxation processes of mono- and bilayer domains 
of liquid crystalline Langmuir films on water. Langmuir, 12(23), $5630-5635$.

Li, X. J., \& Schick, M. (2001). Theory of tunable pH-sensitive vesicles of anionic and cationic lipids or anionic and neutral lipids. Biophysics Journal, 80(4), 1703-1711.

Lissant, K. J. (1966). The geometry of high-internal-phaseratio emulsions. Journal of Colloid and Interface Science, 22(5), 462-468.

Lissant, K. J., \& Mayhan, K. G. (1973). Study of medium and high internal phase ratio water-polymer emulsions. Journal of Colloid and Interface Science, 42(1), 201-208.

Matsen, M. W. (1995). Stabilizing new morphologies by blending homopolymer with block copolymer. Physical Review Letters, 74(21), 4225-4228.

Matsen, M. W., \& Schick, M. (1994). Stable and unstable phases of a diblock copolymer melt. Physical Review Letters, 72(16), 2660-2663.

Matsuyama, A., \& Tanaka, F. (1990). Theory of solvation-induced reentrant phase separation in polymer solutions. Physical Review Letters, 65(3), 341-344.

McClements, D. J., \& Demetriades, K (1998). An integrated approach to the development of reduced-fat food emulsions. Critical Reviews in Food Science and Nutrition, 38(6), 511-536.

Mezzenga, R., Fredrickson, G. H., \& Kramer, E. J. (2003). Tailoring morphologies in polymeric high internal phase emulsions by selective solvent casting. Macromolecules, 36(12), 4457-4465.

Mezzenga, R., Folmer, B. M., \& Hughes, E. (2004). Design of double emulsions by osmotic pressure tailoring. Langmuir, 20(9), 3574-3582.

Mezzenga, R., Lee, W. B., \& Fredrickson, G. H. (2006). Design of liquidcrystalline foods via field theoretic simulations. Trends in Food Science and Technology, 17(5), 220-226.

Mezzenga, R., Meyer, C., Servais, C., Romoscanu, A. I., Sagalowicz, L., \& Hayward, R. C. (2005). Shear rheology of lyotropic liquid crystals: A case study. Langmuir, 21(8), 3322-3333.

Mezzenga, R., Ruokolainen, J., Fredrickson, G. H., \& Kramer, E. J. (2003). High internal phase polymeric emulsion by self-assembly of colloidal systems. Macromolecules, 36(12), 4466-4471.

Mezzenga, R., Ruokolainen, J., \& Hexemer, A. (2003). On the role of block copolymer in self-assembly of dense colloidal polymeric systems. Langmuir, 19(20), 8144-8147.
Mezzenga, R., Ruokolainen, J., Fredrickson, G. H., Kramer, E. J., Moses, D., Heeger, A. J., et al. (2003). Templating organic semiconductors by self-assembly of polymer colloids. Science, 299(5614), 1872-1874.

Mezzenga, R., Schurtenberger, P., Burbidge, A., \& Michel, M. (2005). Understanding foods as soft materials. Nature Materials, 4(10), 729-740.

Montalvo, G., Valiente, M., \& Rodenas, E. (1996). Rheological properties of the $\mathrm{L}$ phase and the hexagonal, lamellar, and cubic liquid crystals of the CTAB/benzyl alcohol/water system. Langmuir, 12(21), 5202-5208.

Muller, M., \& Schick, M. (1998). Calculation of the phase behavior of lipids. Physical Review E, 57(6), 6973-6978.

Pham, K. N., Puertas, A. M., Bergenholtz, J., Egelhaaf, S. U., Moussaid, A., Pusey, P. N., et al. (2002). Multiple glassy states in a simple model system. Science, 296(5565), 104-106.

Pusey, P. N., \& van Megen, W. (1986). Phase behavior of concentrated suspensions of nearly hard colloidal spheres. Nature, 320(6060), 340-342.

Radiman, S., Toprakcioglu, C., \& McLeish, T. C. B. (1994). Rheological studies of ternary cubic phases. Langmuir, 10(1), 61-67.

Romoscanu, A. I., \& Mezzenga, R. (2005). Cross-linking and rheological characterization of adsorbed protein layers at the oil-water interface. Langmuir, 21(21), 9689-9697.

Romoscanu, A. I., \& Mezzenga, R. (2006). Emulsion-templated fully reversible protein-in-oil gels. Langmuir, 22(18), 7812-7818.

Sela, Y., Magdassi, S., \& Garti, N. (1995). Release of markers from the inner water phase of $\mathrm{W} / \mathrm{O} / \mathrm{W}$ emulsions stabilized by silicone-based polymeric surfactants. Journal of Controlled Release, 33(1), 1-12.

Shinoda, W., Namiki, N., \& Okazaki, S. (1997). Molecular dynamics study of a lipid bilayer: Convergence, structure, and long-time dynamics. Journal of Chemical Physics, 106(13), 5731-5743.

Stradner, A., Sedgwick, H., Cardinaux, F., Poon, W. C. K., Egelhaaf, S. U., \& Schurtenberger, P. (2004). Equilibrium cluster formation in concentrated protein solutions and colloids. Nature, 432(7016), 492-495.

Yaghmur, A., de Campo, L., Salentinig, S., Sagalowicz, L., Leser, M. E., \& Glatter, O. (2006). Oil-loaded monolinolein-based particles with confined inverse discontinuous cubic structure ( $\mathrm{Fd} 3 \mathrm{~m})$. Langmuir, 22(2), 517-521.

Weaire, D., \& Phelan, R. (1994). A counter-example to Kelvin conjecture on minimal surfaces. Philosophical Magazine Letters, 69(2), 107-110. 